\title{
Aplicación del modelo de series de tiempo a la prospección del servicio de referencia en línea de la Biblioteca del IIMAS-UNAM
}

\author{
Assessing the online reference service of the IIMAS-UNAM library with a time-series model
}

María del Rocío Sánchez Avillaneda (1) y Ricardo Martínez Bravo (2)

(1) Instituto de Investigaciones en Matemáticas Aplicadas y en Sistemas, UNAM, Circuito escolar s/n, Ciudad Universitaria, México, D. F. 04510, marsa@leibniz.iimas.unam.mx. (2) Centro de Investigación en Matemáticas-CONACYT, Jalisco s/n, Col. Valenciana, Guanajuato 36240, México, jricardo@cimat.mx.

\begin{abstract}
Resumen
Se valida el modelo de series de tiempo en un estudio prospectivo del servicio de referencia en línea vía correo electrónico en términos de su demanda a partir de un ejemplo real, en donde el análisis ayude a conocer la tendencia del servicio sobre la base de datos reales y con el propósito de obtener resultados confiables que no sean puramente especulativos, sino que, a partir de una metodología lo suficientemente sólida, se pueda llegar a una mejor planeación del servicio de referencia en esta modalidad. En los resultados se muestra el modelado de los datos consiguiendo demostrar cómo las técnicas estadísticas pueden ser utilizadas para predecir tendencias del servicio en línea. Este estudio aporta un planteamiento teórico que a futuro pueda coadyuvar en la solución de problemas prácticos, considerando el rumbo de las tendencias del servicio de referencia con la aplicación de métodos estadísticos.
\end{abstract}

Palabras clave: Bibliotecas digitales. Servicio de referencia digital. Series de tiempo.

\section{Introducción}

Las nuevas tecnologías de información y comunicación (TIC) han ocasionado que la sociedad $y$ el mundo de las bibliotecas se encuentren ante una serie de fenómenos nuevos, incluyendo la forma de producir, organizar, acceder y recuperar información. En este sentido, han afectado directamente las formas en que las bibliotecas ofrecen sus servicios digitales. En este caso, se habla del servicio de referencia digital como uno de los fenómenos que muestra mayores expectativas de desarrollo.

Sin embargo, en la sociedad en que vivimos, el riesgo y la incertidumbre ante el futuro aparecen como factores predominantes en el desarrollo de cualquier objeto de estudio; para ello, generalmente se recurre a la elaboración de previsiones que tratan de anticipar la evolución de

\begin{abstract}
In this paper, the time-series model is used to assess the e-mail-based online reference service of the IIMASUNAM library, with the aim of obtaining a prospective of its future trends.
\end{abstract}

Keywords: Digital library. Digital reference service. Time-series model.

algún fenómeno. La literatura muestra claramente que el disponer, en el presente, de un conocimiento sobre el futuro, aunque sea de forma aproximada, facilita la toma de decisiones en las que se incurre cuando se pretende modificar alguna realidad.

Es entonces, en este escenario, donde se encuadra la aplicación del modelo de Series de Tiempo como alternativa metodológica para determinar prospectivas del servicio de referencia digital.

El propósito de realizar un análisis de este tipo consiste en extraer las regularidades que se observan en el comportamiento pasado de una variable, es decir, obtener el mecanismo que la genera, y conocer sobre la base de ello, su comportamiento en el tiempo, y bajo el supuesto de que las condiciones estructurales que con- 
forman el fenómeno objeto de estudio permanezcan constantes, entonces predecir el comportamiento futuro reduciendo la incertidumbre en la toma de decisiones.

Si bien es cierto que las aplicaciones son amplias, existen estudios en el campo bibliotecológico que han utilizado el modelo de series de tiempo para conocer pronósticos, tal como el del autor William G. Jones (1973) A time-series simple approach for measuring use in a small library, trabajo que describe una investigación realizada en la biblioteca del Institute for Social Research (ISR) de la University of Michigan para conocer el uso de los servicios considerando como parámetros el número de usuarios que la utilizan, de tal manera que se logra obtener una buena estimación del uso total por hora, día, semana, mes, trimestre y año. En esta línea de investigación también aparece el trabajo de Crawford, S. (1981) enfocado al análisis de series de tiempo en bibliotecas especializadas Health sciences libraries in the united states. A time series analysis, 1969-1979. Y dos trabajos más aplicados en bibliotecas académicas, el primero sustentado por Brooks, T. A. (1984) Using time-series regression to predict academic library circulations el cual describe la utilización de cuatro métodos para pronosticar los promedios mensuales de la circulación en 15 bibliotecas. Para ello, presenta los resultados que demuestran la utilidad de la aplicación de los métodos del pronóstico que pueden modelar los ciclos de las estadísticas académicas de la biblioteca. $Y$ el segundo publicado por Naylor, M. y Walsh, K. (1994) titulado "A time-series model for academic library data using intervention analysis" estudio que presenta un modelo de series de tiempo usando datos semanales y analizados a través del paquete estadístico SPSS (1).

Como se puede observar, se ha adoptado el modelo de series de tiempo como un método para la obtención de pronósticos de actividades específicas, tal como el uso de la biblioteca o la circulación del material utilizando parámetros temporales como horas, semanas, meses o años. Así, la aplicación del análisis de tiempo ayudará en gran medida a anticipar los sucesos que están por venir y ofrecer diferentes posibilidades de futuros alternativos que pueden ser investigados para después optar por el más conveniente.

Sin duda alguna, el modelo a seguir presenta importantes ventajas sobre las formas convencionales de predicción de resultados de acciones y decisiones; es decir, permite interpretar y predecir las dinámicas y controles en la toma de decisiones, pero, sobre todo, porque es factible para encontrar una tendencia de cierto fenómeno, en este caso, la demanda del uso del servicio de referencia en línea a través del correo electrónico en una biblioteca académica.

\section{El servicio de referencia digital}

"La biblioteca digital es un sistema de información en red que requiere de tecnologías específicas para compartir recursos y ofrecer a sus usuarios contenidos y servicios digitales, cuya información y medios de comunicación se encuentran en servidores distribuidos en diferentes latitudes del mundo" (Torres, 2005). En esta noción de biblioteca los servicios son el resultado del manejo de la información digital a través de tecnologías de la comunicación y de la información, lo que permite características distintas de los servicios que se ofrecen en la biblioteca tradicional. Tal es el caso del servicio de referencia digital como una nueva forma de ofrecer información y responder a las demandas de la comunidad en un ambiente en línea.

Existen diversos estudios referidos a los servicios en la biblioteca digital (Sloan, 1998), y en particular al servicio de referencia como parte integral de todo el conjunto de servicios bibliotecarios que la biblioteca ofrece a determinada comunidad de usuarios. Este aspecto se aborda claramente en el ámbito anglosajón desde una perspectiva teórica y práctica; es decir, disertaciones que han pretendido mostrar aquellos aspectos que se han relacionado con el servicio y sus respectivas implicaciones. Sin embargo, pese a lo numeroso de este tipo de estudios, en casi todos los casos se ejemplifica a partir de los intentos que se están haciendo para poner en marcha servicios de consulta. Muchas de las aportaciones recientes se pueden obtener a través del portal de ACM Digital Library a partir de las experiencias de la Virtual Reference Desk y AskEric, con especial enfoque a la creación de servicios de información en Internet para construir sistemas operacionales de referencia digital.

De esta manera, existen documentos y sitios web dedicados al análisis y elaboración de herramientas de software de referencia digital, otros más enfocados a establecer políticas y procedimientos de referencia para diseñar un servicio de esta naturaleza; así como la identificación de parámetros aplicables tanto para el servicio en modo síncrono como asíncrono (2) $y$, sin dejar a un lado el estudio de usuarios, tipo de preguntas y respuestas, y los mejores sistemas para implementarlo.

Mientras, en el contexto latinoamericano también existen estudios concretos que muestran 
los aspectos de carácter analítico y descriptivo del servicio de referencia tradicional, considerando su concepto, sus características y, por supuesto, el surgimiento de una nueva práctica reconocida como servicio de referencia digital. Sin embargo, y específicamente en México, no existe ningún estudio prospectivo con mayor profundidad que pueda decir hacia dónde se dirige el servicio de referencia actual y cuáles son las tendencias de desarrollo.

Al respecto, cabe mencionar que aunque este tipo de servicio aparece con diferentes expresiones - referencia electrónica, referencia en tiempo real, referencia en vivo, referencia en línea, referencia virtual-, en la actualidad el término más utilizado tanto en documentos científicos como en eventos académicos a nivel internacional es el de servicio de referencia digital.

Es así, que se habla del servicio como aquél que complementa las actividades básicas de la biblioteca con un servicio de referencia mucho más especializado y rápido a través de una computadora (Internet y sistemas expertos de preguntas/respuestas), proporcionando mediación humana en línea con el propósito de identificar y resolver problemas de información (Lankes, 2004).

En un concepto más amplio, Lifeng Han (2003) menciona que los servicios de referencia digital terminológicamente representan a aquellos servicios proporcionados a través de medios electrónicos haciendo uso de aquellos de tipo asincróno (correo electrónico, formularios-web) y sincróno (chat, mensajería instantánea, videoconferencias, y otros en tiempo real). Entretanto, en un sentido estricto, la referencia digital es usualmente utilizada como un sinónimo de referencia electrónica en tiempo real. De esta manera, el autor señala que los servicios de referencia tradicional se realizan cara a cara o contacto directo a través de teléfono o fax dentro de la biblioteca, mientras que con el servicio de referencia digital los usuarios pueden tener acceso a los servicios a pesar del tiempo y la distancia, siendo éste último un servicio que brinda más alternativas y flexibilidad a los usuarios.

Dentro del contexto de biblioteca digital ésta utiliza las computadoras para el almacenamiento y la comunicación, así como máquinas conectadas en red que emulan, reproducen y extienden los servicios que proporciona a la biblioteca tradicional, en este caso el servicio de referencia digital. Es por ello, que la biblioteca digital ofrece el mismo servicio, sólo que a través del empleo de herramientas tecnológicas que facilitan el almacenamiento, la búsqueda y la recupe- ración. Es decir, es una actividad que en esencia no ha tenido cambios: el objetivo primordial es ayudar al usuario en cualquier forma y medio. Al respecto, únicamente se ha ampliado la manera de proporcionar dicho servicio a través de los recursos que se tengan disponibles.

\begin{tabular}{ll}
\hline Alcances & Limitaciones \\
\hline $\begin{array}{l}\text { Acceso remoto con interfaz } \\
\text { persigable a servicios }\end{array}$ & $\begin{array}{l}\text { Requerimiento de clave } \\
\text { personal o institucional } \\
\text { (password) }\end{array}$ \\
\hline $\begin{array}{l}\text { No existen límites de tiempo } \\
\text { y espacio }\end{array}$ & $\begin{array}{l}\text { Restricciones del servicio } \\
\text { acorde con políticas } \\
\text { institucionales }\end{array}$ \\
\hline $\begin{array}{l}\text { Acceso a información en } \\
\text { línea y con la opción de } \\
\text { visualizar, imprimir y } \\
\text { guardar }\end{array}$ & $\begin{array}{l}\text { Información protegida por } \\
\text { derechos de autor (copyright) }\end{array}$ \\
\hline $\begin{array}{l}\text { Acercamiento constante } \\
\text { entre usuario y referencista }\end{array}$ & $\begin{array}{l}\text { No existe interacción cara a } \\
\text { cara por lo que se complica la } \\
\text { entrevista entre ambos }\end{array}$ \\
patrones
\end{tabular}

Tabla I. Servicio de referencia digital: alcances y limitaciones

En términos generales, los alcances y limitaciones se delimitarán por el aspecto tecnológico y profesional; ambos factores podrían ayudar a lograr un nuevo avance en el ámbito bibliotecológico (Tabla I). Finalmente, se puede comprender que el paso del servicio tradicional de referencia al servicio de referencia digital no es algo inédito, sino simplemente es algo complementario a lo ya existente. "En este sentido no puede hablarse de un nuevo servicio sino del mismo, pero bajo procesos que permiten mayor amplitud" (ibidem, p. 43).

Sin embargo, el presente estudio no pretende llevar a cabo revisiones conceptuales del término, sino que su objetivo principal es demostrar que el uso y aplicación del modelo de series de tiempo es factible para determinar prospectivas del servicio de referencia en línea a través de alguno de los medios de comunicación, como correo electrónico, chat o formulario web.

\section{Enfoque prospectivo}

Desde la antigüedad, el hombre ha sentido la necesidad de conocer el futuro, y de prever lo que va a suceder haciendo uso de ciertos métodos como la magia que, mediante algunas prácticas, pretendía adivinar lo que iba a suceder. En cambio, hoy en día la situación es muy distinta, pero más en cuanto a los métodos que 
en cuanto a la necesidad percibida por el hombre.

De esta manera, los métodos actuales, con un enfoque absolutamente racionalista y en muchos casos matemático-estadístico, buscan la respuesta a la misma pregunta de siempre: ¿cómo será el futuro?.

En este sentido, la noción de la propia naturaleza del futuro ha cambiado, y ha pasado de ser algo sometido al libre albedrío de fuerzas sobrenaturales (idea base de los métodos mágicos) a ser esencialmente el resultado del pasado y del presente, con una componente impredecible (aleatoria) más importante cuanto más se adentra en el futuro.

Ya en el siglo XIV, Nicolás Maquiavelo, enfoca la cuestión con una acertada frase: "Para predecir lo que ha de suceder, hay que observar antes lo que ha ocurrido anteriormente." Aquí está la clave de la técnica que será estudiada en el presente trabajo, es decir el futuro como consecuencia del pasado. En esta línea, y más recientemente, el Marques de Halifax afirmaba que "La mejor cualidad de un profeta es la buena memoria", donde la percepción de tal realidad es de gran importancia, pues de ella se sigue que el análisis y modelización de los hechos pasados va a permitir hacer previsiones sobre el futuro.

La prospectiva, como tal, busca, de manera científica, anticipar los sucesos que están por venir y ofrecer diferentes posibilidades de futuros alternativos que pueden ser investigados para después optar por el más conveniente. Es así, que la postura de la prospectiva hacia el futuro es eminentemente proactiva, su enfoque se orienta a la idea de que el futuro es sujeto de creación, pues la posibilidad de que algo ocurra es directamente proporcional a la actividad que se despliegue para que así sea, sin embargo, dice el autor, que no es inoportuno conocer las tendencias, pues el futuro juega en el presente, pero no hay que olvidar que el impacto de la retrospectiva, es la herencia del pasado (Majul, 2003).

\subsection{La previsión proyectiva}

La previsión proyectiva se basa en la información histórica para que mediante su análisis se logren identificar pautas de comportamiento que sigan siendo válidas en futuro. Para ello, se requiere la hipótesis de mantenimiento del entorno y, poder asegurar la validez de ésta sólo a corto plazo.

La información que generalmente manejan las técnicas proyectivas tiene la forma de las llama- das series temporales, que son sucesiones de valores referidos a una misma variable y correspondiente a distintos instantes de tiempo.

Ejemplo de series temporales son los casos declarados de cáncer en México mes a mes; la ventas de una empresa por trimestres; y, más cercano al objetivo del presente estudio, el número de consultas de referencia en una biblioteca en un periodo determinado.

Al respecto, los métodos clásicos parten de un enfoque determinista, ya que la serie temporal sigue una pauta de comportamiento fija, en la que las desviaciones observadas son sólo el efecto de hechos puntuales ocurridos en el entorno sin que esos hechos afecten a la pauta base del modelo.

Dentro de este tipo de métodos, se pueden citar los siguientes: métodos de proyección de tendencias -identificación de tendencias (ajustes de regresión) y métodos suavizados-, y métodos de descomposición de series temporales, donde se utilizan los métodos basados en el suavizado y la descomposición de series cronológicas.

\subsection{Pronósticos}

Es un método mediante el cual se intenta conocer el comportamiento futuro de alguna variable con algún grado de certeza. Es una estimación anticipada del valor de una variable, por ejemplo: la demanda de algún producto. Para ello, existen tres grupos de métodos de pronósticos: cualitativos, proyección histórica y los causales.

Se diferencian entre sí por la precisión relativa del pronóstico del largo plazo en comparación con el de corto plazo, el nivel de herramientas matemáticas requerido y la base de conocimiento como sustrato de sus proyecciones.

Los beneficios del pronóstico son apoyar la toma de decisiones proporcionando información congruente y exacta, la cual se calcula utilizando modelos matemáticos de pronóstico, datos históricos del comportamiento del fenómeno y el juicio del personal involucrado en la institución.

Asimismo, permite gran flexibilidad en la elaboración de pronósticos y para la creación y comparación de múltiples escenarios para efectos de análisis de servicios proyectados (por ejemplo, el servicio de referencia en línea en una biblioteca), apoyando las decisiones de una manera eficaz y oportuna; esto, al pronosticar los lineamientos de los servicios y las demandas establecidas.

Dentro de las características de los pronósticos, está aquella que específicamente trata con el 
futuro y el tiempo, y que ambos se encuentren directamente involucrados. El segundo elemento es la incertidumbre, que siempre está presente. $\mathrm{Y}$ el tercer elemento, presente en grado variable en todas las situaciones descritas, es la confianza de la persona que hace el pronóstico sobre la información contenida en datos históricos. En este sentido, se tiene la siguiente pregunta: ¿qué tan importante es el pasado para estimar el futuro? La respuesta implica conocer si el patrón que ha ocurrido en el pasado se repetirá en el futuro.

Existen ciertos métodos y técnicas para determinar pronósticos; entre ellos, los métodos cuantitativos: el análisis de series de tiempo, el cual consiste en encontrar el patrón del pasado y proyectarlo al futuro. Éste a su vez tiene patrones como: la tendencia, el efecto estacional, el efecto cíclico, y las variaciones irregulares, los cuales serán definidos más adelante.

Por otro lado, cabe mencionar que los métodos de proyección tratan de encontrar el patrón real de los datos para proyectarlos al futuro a través de la aplicación de: (a) los promedios móviles, y (b) la suavización exponencial.

El promedio móvil sirve para pronosticar los valores de datos del siguiente periodo de la serie de tiempo, pero no de los datos de periodos más distantes a futuro. Es, por lo tanto, un método adecuado de pronóstico cuando en los datos no está presente la influencia de una tendencia, un efecto cíclico o estacional. Así, este procedimiento es de gran utilidad para promediar el componente irregular de los datos más recientes de una serie.

La suavización exponencial es un método de pronóstico basado también en el uso de promedios móviles, y dado que para determinar dicho pronóstico para el siguiente periodo debe disponerse del valor más reciente de la serie de tiempo, la suavización exponencial sólo puede usarse para pronosticar el valor para el periodo de la siguiente en la serie de tiempo, pero no para varios periodos futuros (Kazmier, 2000).

\subsection{Análisis de series temporales}

El análisis de series de tiempo es el procedimiento por el cual se identifican y aíslan los factores relacionados con el tiempo que influyen en los valores observados en las series; y, una vez identificados, estos factores pueden contribuir a la interpretación de valores históricos y a pronosticar valores futuros. Por ello, una de las técnicas adecuadas para hacer inferencias sobre el futuro es el análisis de series de tiempo, ya que además es un tipo de inferencia estadís- tica cuantitativa que se hace acerca del futuro de alguna variable o compuesto de variables basados en sucesos pasados.

Se le llama serie de tiempo a un conjunto de mediciones de cierto fenómeno registradas de forma secuencial en el tiempo. Esto es, dada una serie:

$$
\begin{aligned}
& \{x(t 1), x(t 2), x(t 3), \ldots x(t n)\} \text { donde, } \\
& x=\text { representa una variable aleatoria, } y \\
& t_{n}=\text { representa la correspondiente observa- } \\
& \text { ción de dicha variable, }
\end{aligned}
$$

tiene por objeto describir el comportamiento de la serie y buscar posibles patrones temporales que permitan sobrepasar la incertidumbre del futuro (Arellano, 2001).

Una serie de tiempo es un conjunto de valores observados durante una serie de periodos temporales secuencialmente ordenada; y se representa por medio de una gráfica de líneas, sobre cuyo eje horizontal se representan los periodos y en cuyo eje vertical se representan los valores de la serie (ibidem, p. 282).

Así, una serie temporal se puede definir como una sucesión de observaciones correspondientes a una variable en distintos momentos de tiempo, donde las series pueden tener una periodicidad anual, semestral, mensual, trimestral, etc., según los periodos de tiempo en que vengan recogidos los datos que la componen (Figura 1).

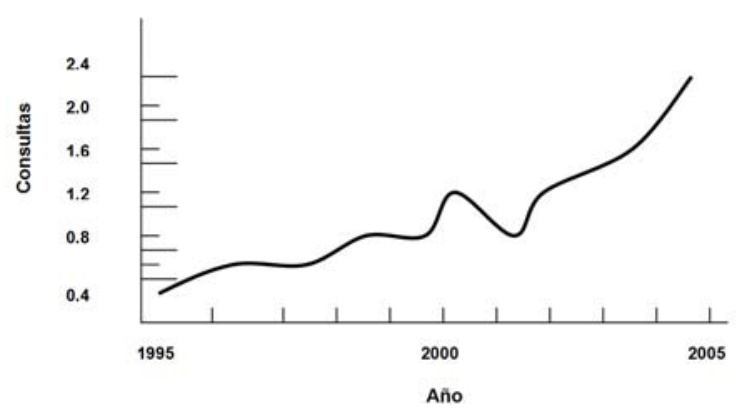

Figura 1. Gráfica de líneas de una serie temporal

El análisis de series de tiempo o cronológicas puede, por tanto, hacerse con un doble propósito: (a) describir la evolución que la serie ha tenido en el pasado y (b) predecir sus valores respecto a un futuro más o menos cercano (Rodríguez, 2000).

Desde la perspectiva de Luis M. Molinero (2004), los objetivos del análisis de series temporales son diversos, pudiendo destacar la predicción, el control de un proceso, la simulación 
de procesos, y la generación de nuevas teorías; considerando así que la predicción es la estimación de valores futuros de la variable en función del comportamiento pasado de la serie. Así, por ejemplo, la predicción mediante modelos basados en la teoría de series temporales, puede servir para una buena planificación de recursos, en función de la demanda que se espera en el futuro prevista por el modelo.

En la teoría de control de procesos, se trata de seguir la evolución de una variable determinada con el fin de regular su resultado. La simulación se emplea en investigación aplicada, cuando el proceso es muy complejo para ser estudiado de forma analítica.

\subsubsection{Componentes de una serie temporal}

La metodología tradicional para el estudio de series temporales se basa fundamentalmente en descomponer las series en varias partes (Kazmier, op. cit., p. 314). Es decir desde una perspectiva teórica y con el objeto de comprender mejor la evolución de un determinado fenómeno, el enfoque clásico o de descomposición de series temporales considera que el comportamiento de una variable en el tiempo es el resultado de la integración de cuatro componentes fundamentales: tendencia, fluctuaciones cíclicas, variaciones estacionales, y variaciones irregulares.

Tendencia. Llamada también movimiento de gran duración e incluso trend utilizando la palabra inglesa. Se considera tendencia al movimiento suave y regular de la serie a largo plazo. Es una componente que reviste gran interés ya que refleja la dirección del movimiento de una determinada variable. De esta forma puede detectarse si, a largo plazo, la serie adopta una marcha persistente, ya sea de crecimiento, decrecimiento o estabilidad, aunque para observar esto es necesario disponer de un horizonte temporal amplio. La predicción de esa componente suele ser en muchos casos el objetivo del análisis de series temporales a través de los modelos de ajuste de tendencia, donde se supone que la serie carece de variaciones estacionales y cíclicas.

En este caso y dentro del análisis de la tendencia, los medios más utilizados para detectar y eliminar la tendencia de una serie se basan en la aplicación de filtros a los datos. Un filtro no es más que una función matemática que aplicada a los valores de la serie produce una nueva serie con unas características determinadas. Entre esos filtros se encuentran las medias móviles o también conocidas como promedios móviles.
Un promedio móvil es el promedio de los (n) valores de datos más recientes de una serie de tiempo. Este procedimiento puede representarse como sigue:

$$
P M=\frac{\sum(n)}{n}
$$

donde:

- $\quad P M=$ Promedio móvil

- $\Sigma=$ Suma de los $\mathrm{n}$ valores más recientes

- $n=$ Total de datos

sirve para pronosticar los valores de datos del siguiente periodo de la serie de tiempo, es por lo tanto, un método adecuado de pronóstico cuando en los datos no está presente la influencia de una tendencia cíclica o estacional.

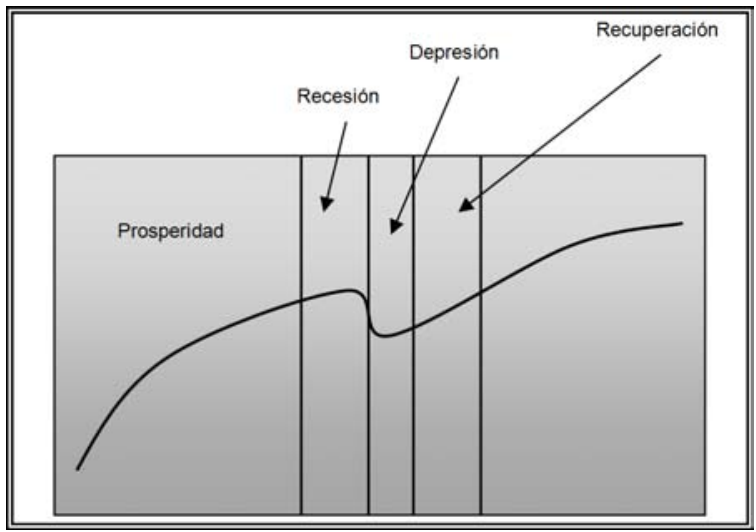

Figura 2. Las fases de un ciclo.

Fluctuaciones cíclicas. En las series, sobre todo en las económicas, la tendencia puede disgregarse a su vez en un factor de tendencia pura, que representa la evolución suave y continua a largo plazo, y un factor de tipo oscilante, caracterizado por movimientos recurrentes en torno a la tendencia, que se repiten cada varios años, y al que se le denomina ciclo económico. Un ciclo se distingue, pues, por una serie de movimientos ascendentes y descendentes separados por puntos de inflexión que en la terminología económica corresponden a las denominadas fases de recuperación, prosperidad, recesión y depresión, tal como se muestra en la Figura 2.

Estacionalidad. En la representación gráfica de muchas series se observa un patrón sistemático que se repite todos los años, es decir, la serie, por ejemplo, presenta siempre una disminución de valor en algún mes concreto. Si se detecta este hecho, entonces se tendrá la seguridad de que la serie está afectada de estacionalidad.

La estacionalidad se define como aquellos movimientos regulares de la serie que tienen una periodicidad inferior al año. En este sentido, 
recogería, por tanto, las oscilaciones que año con año se repiten en una serie de forma periódica.

Existen muchos casos de series donde se observa claramente esta componente y la mayor parte de las veces obedece a factores institucionales o climatológicos. Por ejemplo, en una biblioteca, el hecho de que los periodos vacacionales se concentren en los meses de verano e invierno son factores que condicionan los valores de las series relacionadas con los servicios que se proporcionan, y esto no significa que pueda afectar directamente en la calidad del servicio, sino que, por un mero factor institucional, año tras año se irá repitiendo el mismo fenómeno.

En este caso, si no se tuviera en cuenta este detalle y se considerara la decisión obviando que la serie presenta estacionalidad, seguramente las predicciones serían erróneas. Muchas veces, el estudio de este componente puede constituir la finalidad del trabajo de investigación, ya que su conocimiento resulta de gran trascendencia en numerosos fenómenos que están afectados por la estacionalidad y puede favorecer actuaciones tendentes a modificar algún comportamiento determinado (Chatfield, 2003).

Componente irregular. Se caracteriza porque no responde a un comportamiento sistemático o regular y, en consecuencia, no sería posible su predicción. En la gráfica, está representado por un pico irregular $u$ observaciones anormales y que se sabe que la causa que los provocó no es de esperar que influya en el futuro de la serie; por ello, es conveniente eliminarlos, o no tenerlos en cuenta, con el fin de que factores esporádicos no distorsionen los resultados del análisis. Por ejemplo, dichos factores pueden ser inundaciones, terremotos, incendios, accidentes, huelgas, etc.

Con el análisis de series temporales se pretende encontrar las pautas de regularidad que sigue cada componente a fin de reproducir el comportamiento de la serie. Es decir, se trata de extraer datos que permitan interpretar de una manera más correcta lo que ha sucedido con la variable objeto de estudio a lo largo del tiempo, sin las distorsiones o interferencias provocadas por el componente irregular. De esta manera, se estará en mejor condición para efectuar una predicción de los valores futuros de la serie.

La componente irregular se obtendría, en el análisis clásico, como parte residual una vez estimadas y eliminadas las demás componentes. Para ello, existen fórmulas que permiten la suavización (aislamiento de comportamientos) mediante el empleo de promedios.

Por último, es importante aclarar que en una serie de tiempo no tienen por qué estar presentes todas las componentes anteriormente mencionadas (tendencia, ciclo, estacionalidad e irregularidad), por lo que es de suma importancia analizar cada parte de la serie con la finalidad de obtener los resultados más reales posibles y sobretodo conocer la tendencia del objeto de estudio en cuestión (Morice, 1974).

\subsubsection{Aplicaciones y utilidad}

\section{Según Swoboda (1975),}

Las series de tiempo se pueden encontrar con frecuencia y facilidad en periódicos y revistas de todo tipo, identificadas en forma de curvas más o menos originales o raras que suben o bajan, y son no necesariamente reconocidas por especialistas en estadística. También es una serie en el tiempo el cambio de clima que se produce en una reunión de trabajo, y si este tipo de estadística es tan frecuente deber haber una razón. La serie en el tiempo aporta un elemento dinámico a la estadística; no sólo permite conocer el transcurrir del tiempo hasta el momento, sino que estimula al espectador a imaginarse nuevos modelos para el futuro, y aunque no puedan dar visiones futuristas y fantásticas, entusiasman como coeficientes o distribuciones de frecuencias estacionarias, que ponen en juego la dimensión del tiempo y que a la vez muestran con pocas líneas o números, procesos que nunca se hubiesen supuesto o temido, ni tan sólo intuido.

El análisis de series de tiempo es usado para muchas aplicaciones, tales como pronósticos económicos, pronóstico de ventas, análisis presupuestario, proyecciones de producción, proceso y control de calidad, estudios de inventario, proyecciones de carga de trabajo, análisis de censos y, en general, cualquier aplicación donde se requiera predecir o conocer la evolución de algún fenómeno (NIST/SEMATECH, 2007).

Por ejemplo, el uso de la Internet como uno de los fenómenos de gran auge, en el cual se puede estudiar la forma de acceso considerando básicamente el factor ancho de banda. De tal forma, que se podría medir tanto el avance tecnológico en función de la forma y características con que se accede a la red, así como el conocer cuáles son las velocidades de comunicación, cuántos usuarios hay y a qué nivel de penetración se llega el uso de la Internet. En este sentido, se puede determinar la tendencia en la cantidad de usuarios con conexiones a Internet.

Por esta razón, la aplicación del análisis de tiempo ayudará en gran medida a anticipar los sucesos que están por venir y ofrecer diferentes posibilidades de futuros alternativos que pueden 
ser investigados para después optar por el más conveniente.

\section{Desarrollo del estudio}

El interés por estudiar el servicio de referencia digital reside principalmente en presentar la característica de ser un servicio novedoso, por lo que es importante plantearse propuestas para determinar el desarrollo a futuro de este tipo de servicios. Ahora bien, para determinar el desarrollo ideal de estos servicios es necesario conocer las tendencias que se perfilan desde la práctica.

La aplicación del modelo de Series de Tiempo a este tipo de servicio se justifica si se considera que éste, por su aparición tan reciente, requiere de métodos que permitan hacer una prospección confiable con datos derivados de su uso inicial.

En esta dirección, cabe señalar que existen numerosos estudios sobre el tema del servicio de referencia digital, sin embargo, la mayoría de ellos se enfocan a investigaciones puramente especulativas sobre el escenario probable del servicio, es decir, aportaciones de lo que podría suceder en algunos años, pero estas ideas son supuestos que no se basan en datos reales. Por lo que es clara la falta de estudios que, con una base metodológica lo suficientemente sólida, ayuden al conocimiento de las tendencias del servicio de referencia digital.

Al respecto, cabe señalar que los estudios realizados en relación con el desarrollo del servicio de referencia en el contexto digital, carecen de una metodología que ayude a determinar desde la práctica el camino que sigue el desarrollo del servicio. Es decir, carecen de un método que permita conocer hacia dónde se dirige el servicio actual a partir de datos temporales validados por el modelo de Series de Tiempo.

Por ello, se trata de demostrar la utilidad del modelo, a partir de su aplicación en el estudio de uno de los servicios digitales que más desarrollo promete.

En este sentido, en el presente estudio se realizará la validación del método cuantitativo a través de la técnica de Series de Tiempo en la Biblioteca del Instituto de Investigaciones en Matemáticas Aplicadas y en Sistemas de la Universidad Nacional Autónoma de México (IIMAS-UNAM) con servicio de referencia en línea sólo para el caso del uso del correo electrónico.

\subsection{Objetivo y metodología}

En primer instancia, el objetivo es validar el modelo de series temporales en el estudio prospectivo del servicio de referencia vía correo electrónico, considerando como indicador el número de consultas en 2007, de tal manera que se determine el valor futuro que tomará la variable objeto del análisis aplicando dicho modelo; y, por último, conocer la tendencia del servicio por este medio electrónico. Es así, que en la Tabla II se tienen los datos siguientes:

\begin{tabular}{lc}
\hline Mes & Correos \\
\hline Enero & 9 \\
\hline Febrero & 12 \\
\hline Marzo & 10 \\
\hline Abril & 5 \\
\hline Mayo & 14 \\
\hline Junio & 25 \\
\hline Julio & 10 \\
\hline Agosto & 22 \\
\hline Septiembre & 30 \\
\hline Octubre & 24 \\
\hline Noviembre & 40 \\
\hline Diciembre & 25
\end{tabular}

Tabla II. Datos de la serie original del servicio de referencia vía Correo electrónico

Para efectos de este estudio, se plantean las preguntas siguientes: ¿Cómo se obtendrá la tendencia del servicio de referencia en línea a través del correo electrónico? ¿Cómo se obtienen las predicciones (pronóstico) para este servicio?

La respuesta a la primera pregunta se logrará por medio de dos métodos a aplicar: (A1) los promedios móviles, y (A2) el ajuste lineal

Así, se tienen los siguientes datos reales:
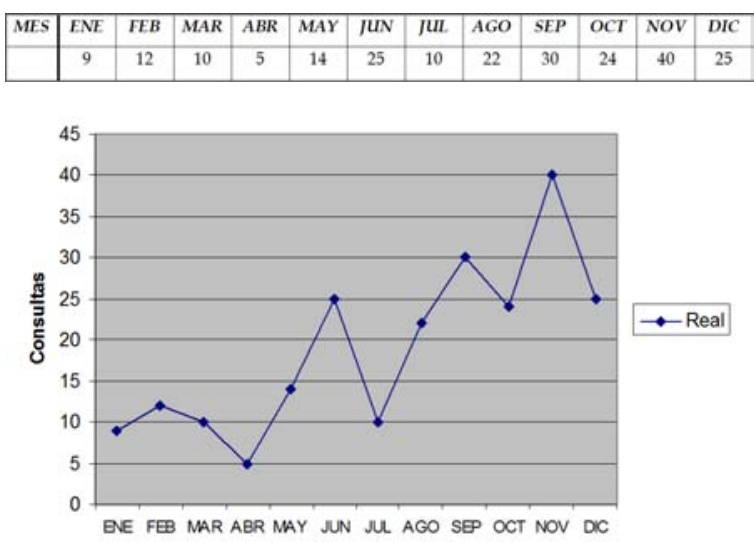

Figura 3. Representación de la serie para el servicio de referencia vía Correo electrónico 


\section{A1. Promedios Móviles}

A través de la aplicación del Promedio Móvil de orden q se obtendrá la tendencia, representado por $P M_{(\mathrm{q})}$

$$
\begin{aligned}
& y_{1}=\frac{x_{1}+x_{2}+\cdots+x_{q}}{q} \\
& y_{2}=\frac{x_{2}+x_{3}+\cdots+x_{q+1}}{q} \\
& y_{3}=\frac{x_{3}+x_{4}+\cdots+x_{q+2}}{q}
\end{aligned}
$$

Donde:

$$
\begin{aligned}
& x=\text { son los datos originales por mes } \\
& q=\text { es el orden del promedio móvil (3) } \\
& y=\text { es el dato suavizado por el promedio } \\
& \text { móvil }
\end{aligned}
$$

Previo a conocer la tendencia, se observa que en la Figura 3 aparece un movimiento que es recurrente, es decir un patrón que posiblemente se repetirá en los años subsiguientes, entonces se puede decir que la serie está afectada de estacionalidad.

La estacionalidad está definida como los movimientos regulares de la serie que tienen una periodicidad inferior al año, donde las oscilaciones que año con año se repiten en una serie se presentan de forma periódica. Es decir, estamos hablando de los periodos vacacionales, donde el hecho de que, por costumbre, las vacaciones laborales se concentren en los meses de verano e invierno determina los valores de la serie presentados en el número de consultas recibidas y que se verán reflejados en la atención al usuario en línea.

En definitiva, esta serie presenta sistemáticamente una disminución durante el mes de julio, agosto y diciembre. Empero, no quiere decir que con ello el servicio de referencia que ofrece la Biblioteca-IIMAS-UNAM sea de mala calidad en este periodo, sino que por un factor institucional que se presenta año tras año se repetirá el mismo movimiento en la serie de datos.

Con el fin de eliminar los efectos de estacionalidad se obtendrá la serie suavizada con un promedio móvil de orden (q), en este caso se decidió utilizar este indicador porque es uno de los más adecuados para identificar la tendencia, además de porque permite estimar el promedio de las consultas de un cierto número de periodos arrojando el cálculo del pronóstico del uso del servicio en el futuro.
El valor $q$ se conoce como el orden y el proceso se denota como $\mathrm{PM}(q)$ lo que en inglés significa Moving Average $(\mathrm{MA}(q))$, la fórmula anterior sugiere un promedio aritmético y el efecto de este procedimiento es el de eliminar las fluctuaciones entre cada $q$ periodos, donde la representación del gráfico de la serie se suaviza.

Teniendo como datos filtrados los siguientes y representados de manera gráfica en la Figura 4.

\begin{tabular}{|c|c|c|c|c|c|c|c|c|c|c|c|c|}
\hline MES & 1 & 2 & 3 & $\mathbf{4}$ & 5 & 6 & 7 & 8 & 9 & 10 & 11 & 12 \\
\hline & & & 10.33 & 9.00 & 9.67 & 14.67 & 16.33 & 19.00 & 20.67 & 25.33 & 31.33 & 29.67 \\
\hline
\end{tabular}

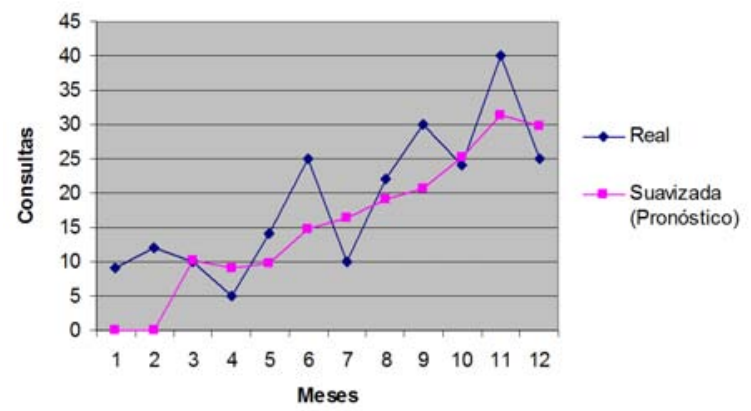

Figura 4. Representación de la serie suavizada

Una vez que realizado el suavizado de los datos de la serie de tiempo de la gráfica, se observa una tendencia de crecimiento lineal. Por lo tanto, se puede hacer el ajuste a una recta, que se presenta en el apartado siguiente:

\section{A2. Ajuste Lineal}

El ajuste se hará por medio del método de mínimos cuadrados (Spiegel, 2001) que ayude a determinar la mejor línea de ajuste y conocer el comportamiento de la tendencia. Por lo tanto, y con base a la ecuación de una recta:

$$
y=b x+a
$$

Los valores $a$ y $b$ están dados por:

$$
\begin{array}{r}
a=\frac{\left(\sum y\right)\left(\sum x^{2}\right)-\left(\sum x\right)\left(\sum x y\right)}{n \sum x^{2}-\left(\sum x\right)^{2}} \\
b=\frac{n \sum x y-\left(\sum x\right)\left(\sum y\right)}{n \sum x^{2}-\left(\sum x\right)^{2}}
\end{array}
$$

Donde:

$$
\begin{aligned}
& x=\text { meses (enero a diciembre). } \\
& y=\text { datos suavizados (ajustados o filtrados) } \\
& \text { a partir del mes de marzo. } \\
& n=\text { número de datos usados (10). }
\end{aligned}
$$


Así, se tienen los datos siguientes:

\begin{tabular}{|c|c|c|c|c|c|c|c|c|c|c|c|c|}
\hline MES & ENE & FEB & MAR & ABR & MAY & JUN & JUL & AGO & SEP & OCT & NOV & DIC \\
\hline Consultas & 9 & 12 & 10 & 5 & 14 & 25 & 10 & 22 & 30 & 24 & 40 & 25 \\
\hline PMovil & & & 10.33 & 9 & 9.67 & 14.67 & 16.33 & 19.00 & 20.67 & 25.33 & 31.33 & 29.67 \\
\hline
\end{tabular}

donde el ajuste lineal se obtendrá usando los datos de la Tabla III.

\begin{tabular}{|c|c|c|c|c|}
\hline$x$ & $y$ & $x 2$ & $x y$ & $y 2$ \\
\hline 3 & 10.33 & 9 & 30.9900 & 106.71 \\
4 & 9.00 & 16 & 36.00 & 81.00 \\
5 & 9.67 & 25 & 48.35 & 93.51 \\
6 & 14.67 & 36 & 88.02 & 215.21 \\
7 & 16.33 & 49 & 114.31 & 266.67 \\
8 & 19.00 & 64 & 152.00 & 361.00 \\
9 & 20.67 & 81 & 186.03 & 427.25 \\
10 & 25.33 & 100 & 253.30 & 641.61 \\
11 & 31.33 & 121 & 344.63 & 981.57 \\
12 & 29.67 & 144 & 356.04 & 880.31 \\
\hline$\sum x=75$ & $\sum y=186.00$ & $\sum x^{2}=645$ & $\sum x y=1609,67$ & $\sum y^{2}=4054.83$ \\
\hline
\end{tabular}

Tabla III. Datos de la serie para el ajuste lineal

$$
\begin{aligned}
& a=\frac{(186)(645)-(75)(1609.67)}{(10)(645)-(75)^{2}}=\frac{-755.25}{825}=-0.9155 \\
& b=\frac{(10)(1609.67)-(75)(186)}{(10)(645)-(75)^{2}}=\frac{2146.7}{825}=2.6021
\end{aligned}
$$

Por tanto si $y=b x+a$ entonces se tiene:

$$
y=2.6021 x-0.9155
$$

Tanto los datos originales como el promedio móvil y el ajuste lineal —este último para obtener la tendencia- (Figura 5).

La tendencia es un movimiento suave y regular de la serie, el cual es una componente de suma importancia ya que refleja la dirección del movimiento de la variable denominada consultas. De esta forma puede detectarse que el servicio de referencia que se ofrece a través del uso del correo electrónico adopta una marcha persistente, es decir, se observa un notable crecimiento, aunque cabe aclarar que para mayor aproximación al comportamiento de la serie es necesario disponer de un horizonte temporal amplio.

Así se tiene para a y b lo siguiente:

\section{Correo Electrónico}

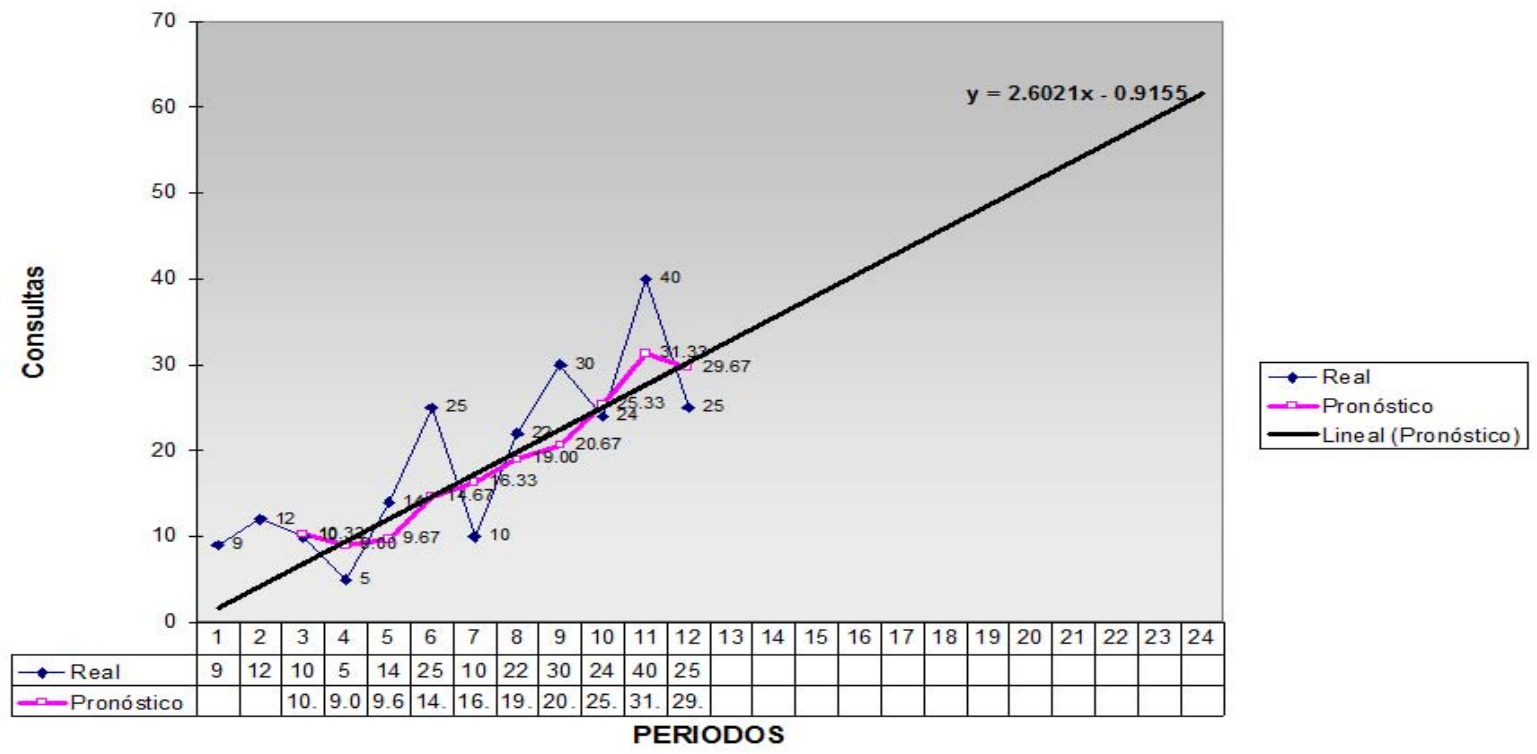

Figura 5. Series de tiempo para el servicio de referencia vía correo electrónico

La respuesta a la segunda pregunta referente a ¿Cómo se obtienen las predicciones (pronóstico) para este servicio? Una vez obtenida la ecuación lineal:

$$
y=2.6021 x-0.9155
$$

$$
\begin{aligned}
& y=2.6021(17)-0.9155 \\
& y=43.32
\end{aligned}
$$

Si se quiere hacer un pronóstico para el mes 17 (mayo 2008), por ejemplo, se tiene que la pre- 
dicción representa un aumento del 42.92\%. Esto es, si en el mes 12 (diciembre 2007) se tiene un estimado de 30.31 consultas, significa que en 5 meses se habrán atendido 13 consultas (Tabla IV).

Cabe aclarar que cuanto más nos alejemos en el tiempo los pronósticos serán menos confiables, por lo que una alternativa al respecto es obtener datos mes a mes y hacer el análisis y cálculos actualizados correspondientes.

\begin{tabular}{|c|c|}
\hline$x$ & $y$ \\
\hline 1 & \\
\hline 2 & \\
\hline 3 & 6.89 \\
\hline 4 & 9.49 \\
\hline 5 & 12.09 \\
\hline 6 & 14.70 \\
\hline 7 & 17.30 \\
\hline 8 & 19.90 \\
\hline 9 & 22.50 \\
\hline 10 & 25.11 \\
\hline 11 & 27.71 \\
\hline 12 & 30.31 \\
\hline 13 & 32.91 \\
\hline 14 & 35.51 \\
\hline 15 & 38.12 \\
\hline 16 & 40.72 \\
\hline 17 & 43.32 \\
\hline 18 & 45.92 \\
\hline 19 & 48.52 \\
\hline 20 & 51.13 \\
\hline 21 & 53.73 \\
\hline 22 & 56.33 \\
\hline 23 & 58.93 \\
\hline
\end{tabular}

Tabla VI. Datos del pronóstico

\section{Resultados y prospectivas futuras}

Esta investigación ha permitido demostrar que el modelo de series de tiempo es una alternativa viable para obtener predicciones confiables y determinar prospectivas del servicio de referencia en línea vía correo electrónico que actualmente se ofrece en la Biblioteca del IIMASUNAM, lo que ha permitido dar respuestas a la interrogante planteada al inicio del presente estudio con especial enfoque a la prospectiva del servicio.

En esta dirección, se cumple con el objetivo de la presente investigación. Es decir, se logró validar el modelo en este servicio, de tal manera que se ha podido observar el comportamiento en el tiempo de las variables analizadas a través de las tendencias estimadas.

En el caso del correo electrónico se observa una tendencia de crecimiento, donde se puede predecir con toda seguridad el comportamiento que tendrá a futuro, induciendo así a la mejor toma de decisiones. En este sentido, aún no es necesario contar con equipo adicional para responder a la demanda de los usuarios; tampoco es ahora prioritario contratar más personal para atender dicho servicio.

Al respecto, cabe señalar que las bibliotecas en general hacen uso del correo electrónico con la finalidad de facilitar la comunicación, específicamente en cuanto a las solicitudes y las transacciones para el préstamo interbibliotecario. De tal forma, que este medio de tipo asíncrono resulta ser una alternativa más que el servicio tradicional (teléfono o cara a cara). De esta manera, el correo electrónico tiene alcances que van más allá de la propia biblioteca y de la individualización. Es decir, permite además establecer una red de comunicación hacia y para un grupo, esto se puede comprobar claramente observando la línea de tendencia (Figura 5) donde la demanda del servicio de referencia por esta vía se está incrementando a la par del uso de Internet.

En términos generales, se puede observar que el impacto tecnológico sobre las bibliotecas ha propiciado que, de nueva cuenta, se redefina el servicio de referencia que actualmente se viene ofreciendo; y la realidad es que en muchos países existen ya servicios plenamente desarrollados y muchos otros a nivel de proyecto, que, sin duda alguna, llegarán a ser útiles y con capacidad de responder a las necesidades de información de usuarios remotos.

En México no se está lejos de esta realidad, ya que se puede hablar del servicio de referencia en entornos digitales gracias al interés que han tenido algunas instituciones por mejorar la calidad de los servicios, pero, sobre todo, por disminuir los límites de tiempo y comunicación geográfica, de tal forma que los usuarios puedan obtener información sin asistir físicamente a la biblioteca. Los avances han sido posibles gracias a la integración de un equipo multidisciplinario, al apoyo institucional y de entidades externas, y a la colaboración con otras instituciones.

La revisión de algunos documentos y sitios han permitido reconocer que tales servicios son nombrados con diferentes términos como consulta electrónica, ayuda en línea, referencia virtual, o bien asesoría virtual. A pesar de ello, cabe señalar que todos estos intentos cubren el 
objetivo de la esencia del servicio, aunque es importante considerar que la tecnología disponible en cada institución dependerá de las herramientas y utilidades que puedan ofrecer a su comunidad.

Al respecto, es indispensable la evaluación de las nuevas tecnologías antes de implementarlas, principalmente porque se ofrecerán servicios de referencia en tiempo real; definitivamente se tiene un mercado cada vez mayor en las opciones de software y hardware lo que a su vez, implica la capacitación, y uso de políticas y estándares del servicio.

La implementación de bibliotecas digitales implica considerar ciertos aspectos en relación a los recursos financieros, de cómputo y sobre todo recursos humanos. De igual manera, es importante tomar en consideración la posibilidad de intercambiar experiencias con otros proyectos que han desarrollado servicios de referencia digital.

Sin duda alguna, hace más de una década que las bibliotecas digitales son consideradas un factor estratégico de competitividad en países desarrollados. Es crítico para todas las entidades involucradas (instituciones de educación superior e investigación) reconocer su importancia y participar activamente en su construcción y aprovechamiento; por tal razón, es significativo pensar en las tendencias del servicio.

En términos generales, los alcances y limitaciones se definirán por el aspecto tecnológico y profesional. Ambos factores pueden ayudar a lograr avances significativos en la construcción de ambientes y herramientas para comunidades que transmiten y generan conocimiento. Para ello, es indispensable definir los aspectos en torno a las habilidades y responsabilidades que deberá asumir el bibliotecario ante esta nueva modalidad de servicio.

\section{Conclusiones}

En el transcurso y desarrollo del presente estudio de investigación, se observa que el modelo de series de tiempo fue adecuado para la descripción y realización de pronósticos (tendencias); en este caso, para el servicio de referencia en línea vía correo electrónico. Sin embargo, una limitante es que sus predicciones sólo son buenas a corto plazo. Por ello, se consideró adecuado un año con datos mensuales. $Y$, tomando en cuenta que un modelo teórico se considera sólo una aproximación a la realidad, se pudo mostrar que a partir de la aplicación de dicho modelo, se logra conocer el desarrollo a futuro del servicio sobre la base de datos reales y confiables, es decir, permite ir más allá de estudios puramente especulativos.

En relación con el objetivo planteado, se logró el modelado de los datos consiguiendo demostrar cómo las técnicas estadísticas pueden ser utilizadas para explicar y predecir aceptablemente los porcentajes de consultas del servicio de referencia en su modalidad de correo electrónico; lo que significa que también puede ser aplicado en aquellas bibliotecas que ofrezcan el servicio a través de formularios-web o chat.

Debe resaltarse que, siempre que sea posible, el modelo debe ser reajustado, para actualizar los pronósticos. Esto es, en la medida en que se posean datos reales posteriores a la fecha del periodo analizado, se deberán calcular nuevamente los parámetros, y así obtener proyecciones actuales, lo cual permitirá realizar programaciones óptimas de los recursos, así como programar mantenimientos de equipo, y ofrecer capacitación y requerimientos de personal sólo cuando sea necesario.

Cabe mencionar que la contribución del presente estudio para la disciplina bibliotecológica es justamente el aporte de un planteamiento teórico que a futuro pueda coadyuvar en la solución de problemas prácticos, considerando el rumbo de las tendencias del servicio de referencia con aplicación de métodos estadísticos.

\section{Notas}

(1) SPSS son las siglas de Statistical Product and Service Solutions. Originalmente SPSS era el acrónimo de Statistical Package for the Social Sciences. Es un completo sistema de análisis de datos que puede generar informes tabulares, gráficos y diagramas de distribuciones y tendencias, así como complejos análisis estadísticos. http://www.spss.com (2008-04-27).

(2) Los sistemas sincrónicos son técnicamente métodos de transmisión digital de datos a velocidades muy altas mediante el uso de circuitos en los que los relojes del emisor y receptor están sincronizados. Se emplean para transferir grandes cantidades de información. El envío y recepción debe ocurrir a unos intervalos de tiempo precisos. La comunicación sincrónica ofrece la posibilidad de intercambiar información desde cualquier sitio, en cualquier momento $\mathrm{y}$, sobre todo, al mismo tiempo; de tal forma, que el usuario y el referencista se entrevistan en tiempo real a través de medios de comunicación basados en Internet y en la tecnología en general. Tipos de comunicación síncrona son: chat, mensajería instantánea, videoconferencia y cámara web, entornos colaborativos y voz sobre IP (Dungan, Frank R. 1998). La comunicación asíncrona se encuentra mediada tecnológicamente y no depende de que el emisor y receptor estén presentes en el mismo tiem- 
po para actuar; ofrece la posibilidad de intercambiar información desde cualquier sitio y en cualquier momento (correo electrónico y formularioweb).

\section{Referencias}

Arellano, M. (2001). Introducción al análisis clásico de series de tiempo. http://www.5campus.com/leccion/seriest (2007-01-14).

Brooks, T. A. (1984). Using time-series regression to predict academic library circulations. // College \& Research Libraries. 45:6 (1984) 501-505.

Chatfield, C. (2003). The analysis of time series: an introducction. London: Chapman and Hall, 2003.

Crawford, S. (1981). Health sciences libraries in the united states: a time series analysis, 1969-1979. // Journal of the American Medical Association. 245:21 (1981) 22372238.

Dungan, Frank R. (1998). Electronic communications systems. Albany: Delmar, 1998.

Han, Lifeng (2003). Information and reference services in the digital library. // Information Services \& Use. 23:4 (2003) 251-262.

International Conference on Digital Libraries: Usability for Digital Libraries. 2002. http://portal.acm.org/dl.cfm (2008-03-17).

Jones, William G. (1973). A time-series simple approach for measuring use in a small library. // Special Libraries. 64:7 (July 1973) 280-284.

Kazmier, Leonard J. (2000). Análisis de series de tiempo y pronósticos económicos. // Estadística aplicada a la administración y a la economía. $3^{a}$ ed. México: McGraw Hill, 2000.

Lankes, R. David (2004). Digital reference. // Encyclopedia of Library and Information Science. New York: Marcel Dekker, 2004. 1-3.

Majul Zamudio, Yazmín. (2003). Planeación prospectiva. México: IPN, Centro de Investigaciones Económicas, Administrativas y Sociales, 2003.

Molinero, Luis M. (2004). Análisis de series temporales. http://www.seh-lelha.org/pdf/tseries.pdf (2008-01-27).

Morice, E. (1974). Diccionario de estadística. México: Continental, 1974.

Naylor, Maiken; Kathlenn, Walsh (1994). A time-series model for academic library data using intervention analysis. // Library \& Information Science Research. 16:4 (1994) 299-314.

NIST/SEMATECH e-Handbook of Statistical Methods. Introduction to time series analysis. http://www.itl.nist.gov/ div898/handbook/pmc/section4/pmc41.htm (2008-0110).

Rodríguez Morilla, Carmen (2000). Análisis de series de tiempo. // Análisis de series temporales. Madrid: La Muralla, 2000.

Sloan, Bernie (1998). Digital reference services bibliography. http://admin2.american.edu/ hodges/index/Bernie\% 20Sloan's\%20VR\%20bibliography.htm (2008-03-15).

Spiegel, Murray R. (2001). Curva de ajuste, regresión y correlación. // Probabilidad y estadística. Bogotá: McGraw-Hill, 2001. 286-289.

SPSS (Statistical Package for the Social Sciences). http://www.spss.com (2008-04-27).

Swoboda, Helmut (1975). Las series de tiempo. // El libro de la estadística moderna. Barcelona: Omega, 1975.
Torres Vargas, Georgina Araceli (2005). La Biblioteca digital. México: UNAM, Centro Universitario de Investigaciones Bibliotecológicas, 2005. 